\title{
Aluthge transformations and invariant subspaces of $p$-hyponormal operators
}

\author{
Muneo $\mathrm{CH}^{*}$ and Tadasi HuRUYA
}

(Received March 4, 2002; Revised April 18, 2002)

\begin{abstract}
It is unknown at present whether every hyponormal operator has a nontrivial invariant subspace. Many authors presented conditions for a hyponormal operator to have nontrivial invariant subspaces. In this paper, we give a $p$-hyponormal version of Nakamura's result [7] by using the principal functions.
\end{abstract}

Key words: hyponormal operator, $p$-hyponormal operator, invariant subspace.

\section{Introduction}

An (bounded linear) operator $T$ on a Hilbert space $\mathcal{H}$ is said to be $p$-hyponormal, if $\left(T T^{*}\right)^{p} \leq\left(T^{*} T\right)^{p}$ for a positive number $p$. If $p=1$, then $T$ is said to be hyponormal, and if $p=\frac{1}{2}$, then $T$ is said to be semihyponormal. We assume that $0<p \leq \frac{1}{2}$. An operator $T$ is called pure if it has no nontrivial reducing subspace on which it is normal.

It is unknown at present whether every hyponormal operator has a nontrivial invariant subspace. Putnam [8] and Apostol and Clancey [2] presented some conditions for a hyponormal operator to have invariant subspaces. Nakamura [7] improved these results. In this paper, we give a $p$-hyponormal version of Nakamura's result.

Let $T=X+i Y$ be a pure hyponormal operator, where $X$ and $Y$ are self-adjoint. Then it is known that $X$ and $Y$ are absolutely continuous (see [4, Chap. 2, Th. 3.2]). For a self-adjoint operator $Z$, let $Z=\int t d G(t)$ be the spectral resolution of $Z$. Then the absolutely continuous support $E_{Z}$ of $Z$ is defined as a Borel subset of the real line (determined uniquely up to a null set) having the least Lebesgue measure and satisfying $G\left(E_{Z}\right)=I$. Then Nakamura's results are as follows.

Theorem A ([7], Theorem 1) Let $T$ be a pure hyponormal operator and $T=X+i Y$ be the Cartesian decomposition of $T$. Suppose that there exists

2000 Mathematics Subject Classification : 47A15, 47B20.

${ }^{*}$ This research is partially supported by Grant-in-Aid Scientific Research (No. 14540190). 
a real $\mu_{0}$ such that the spectrum of $T$ has non-empty intersection with each of the open half-planes $\left\{z: \operatorname{Re} z<\mu_{0}\right\}$ and $\left\{z: \operatorname{Re} z>\mu_{0}\right\}$, and

$$
\int_{E_{X}} \frac{F(x)}{\left(x-\mu_{0}\right)^{2}} d x<\infty
$$

where $F(x)$ is the linear measure of the vertical cross $\sigma(T) \cap\{z: \operatorname{Re} z=x\}$. Then $T$ has a nontrivial invariant subspace.

Theorem B ([7], Theorem 2) In Theorem 1, the existence of a nontrivial invariant subspace is also guaranteed if the integrability condition is replaced by

$$
\int_{E_{X}} \frac{1}{\left|x-\mu_{0}\right|} d x<\infty .
$$

Let $T=U|T|$ be the polar decomposition of $T$. Put $\tilde{T}=|T|^{\frac{1}{2}} U|T|^{\frac{1}{2}}$. Let $\tilde{T}=V|\tilde{T}|$ denote the polar decomposition of $\tilde{T}$. Put $\hat{T}=|\tilde{T}|^{\frac{1}{2}} V|\tilde{T}|^{\frac{1}{2}}$. Then $\tilde{T}$ and $\hat{T}$ are called the Aluthge transformation and the second Aluthge transformation of $T$, respectively. It is well known that if $T$ is $p$-hyponormal, then $\hat{T}$ is hyponormal by [1]. Also it is well known that $\sigma(T)=\sigma(\tilde{T})=$ $\sigma(\hat{T})$.

The main results in this paper are the following:

Theorem 1 Let $T$ be a pure p-hyponormal operator with dense range. For the second Aluthge transformation $\hat{T}$ of $T$, let $\hat{T}=X_{2}+i Y_{2}$ denote the Cartesian decomposition of $\hat{T}$. Suppose that there exists a real $\mu_{0}$ such that the spectrum of $T$ has non-empty intersection with each of the open half-planes $\left\{z: \operatorname{Re} z<\mu_{0}\right\}$ and $\left\{z: \operatorname{Re} z>\mu_{0}\right\}$, and

$$
\int_{E_{X_{2}}} \frac{F(x)}{\left(x-\mu_{0}\right)^{2}} d x<\infty
$$

where $F(x)$ is the linear measure of the vertical cross $\sigma(T) \cap\{z: \operatorname{Re} z=x\}$. Then $T$ has a nontrivial invariant subspace.

Theorem 2 In Theorem 1, the existence of a nontrivial invariant subspace is also guaranteed if the integrability condition is replaced by

$$
\int_{E_{X_{2}}} \frac{1}{\left|x-\mu_{0}\right|} d x<\infty .
$$




\section{Aluthge transformation}

Lemma 3 Let $T=U|T|$ be an operator with $\operatorname{ker}|T|=\{0\}$. If $T$ has a cyclic vector, then the Aluthge transformation $\tilde{T}$ has also a cyclic vector and satisfies $\operatorname{ker}|\tilde{T}|=\{0\}$.

Proof. Let $x$ be a cyclic vector for $T$. For any positive integer $n$,

$$
(\tilde{T})^{n}|T|^{\frac{1}{2}}=\left(|T|^{\frac{1}{2}} U|T|^{\frac{1}{2}} \cdots|T|^{\frac{1}{2}} U|T|^{\frac{1}{2}}\right)|T|^{\frac{1}{2}}=|T|^{\frac{1}{2}} T^{n} .
$$

Let $y$ be a vector such that $\left((\tilde{T})^{n}|T|^{\frac{1}{2}} x, y\right)=0$ for $n=0,1,2, \ldots$ Then

$$
\left(T^{n} x,|T|^{\frac{1}{2}} y\right)=0 \text {. }
$$

Since $x$ is a cyclic vector for $T,|T|^{\frac{1}{2}} y=0$, so that $|T| y=0$. Hence by the assumption we have $y=0$. This implies that $|T|^{\frac{1}{2}} x$ is a cyclic vector for $\tilde{T}$.

Next we show ker $|\tilde{T}|=\{0\}$. Since $\operatorname{ker}|T|=\{0\}$, we may assume that $U$ is isometry. Let $\tilde{T} w=0$, so that $|T|^{\frac{1}{2}} U|T|^{\frac{1}{2}} w=0$. Since $\operatorname{ker}|T|=\{0\}$ and $U$ is isometry, we have $|T|^{\frac{1}{2}} w=0$, that is, $w=0$. Therefore, $\operatorname{ker} \tilde{T}=\{0\}$. Since $\operatorname{ker} \tilde{T}=\operatorname{ker}|\tilde{T}|$, we have $\operatorname{ker}|\tilde{T}|=\{0\}$.

The following lemma improves [3, Lemma 2].

Lemma 4 Let $T=U|T|$ be a pure p-hyponormal operator with dense range. Then the Aluthge transformation $\tilde{T}$ is pure $\left(p+\frac{1}{2}\right)$-hyponormal.

Proof. It is well known that $\tilde{T}$ is $\left(p+\frac{1}{2}\right)$-hyponormal. Hence we may only prove that $\tilde{T}$ is pure. Since $T$ is $p$-hyponormal and has a dense range, $\operatorname{ker} T=\operatorname{ker}|T|=\operatorname{ker} T^{*}=\{0\}$. Hence $U$ is unitary. Let $\mathcal{X}$ be a reducing subspace of $\tilde{T}$ such that $\tilde{T}$ is normal on $\mathcal{X}$. Then for $x \in \mathcal{X}$,

$$
\tilde{T}(\mathcal{X}) \subseteq \mathcal{X},(\tilde{T})^{*}(\mathcal{X}) \subseteq \mathcal{X} \quad \text { and } \quad(\tilde{T})^{*} \tilde{T} x=\tilde{T}(\tilde{T})^{*} x
$$

If $\left((\tilde{T})^{*} \tilde{T}\right)^{n} x=\left(\tilde{T}(\tilde{T})^{*}\right)^{n} x$ for $x \in \mathcal{X}$, then by (1) we have

$$
\begin{aligned}
\left((\tilde{T})^{*} \tilde{T}\right)^{n+1} x & =\left((\tilde{T})^{*} \tilde{T}\right)^{n}\left((\tilde{T})^{*} \tilde{T} x\right)=\left(\tilde{T}(\tilde{T})^{*}\right)^{n}\left((\tilde{T})^{*} \tilde{T} x\right) \\
& =\left(\tilde{T}(\tilde{T})^{*}\right)^{n}\left(\tilde{T}(\tilde{T})^{*} x\right)=\left(\tilde{T}(\tilde{T})^{*}\right)^{n+1} x .
\end{aligned}
$$

Hence we have $\left((\tilde{T})^{*} \tilde{T}\right)^{n} x=\left(\tilde{T}(\tilde{T})^{*}\right)^{n} x$ for every non-negative integer $n$ and $x \in \mathcal{X}$. Since it holds that $f\left((\tilde{T})^{*} \tilde{T}\right) x=f\left(\tilde{T}(\tilde{T})^{*}\right) x$ for every polynomial $f$, we have

$$
\left(|T|^{\frac{1}{2}} U^{*}|T| U|T|^{\frac{1}{2}}\right)^{p+\frac{1}{2}} x=\left(|T|^{\frac{1}{2}} U|T| U^{*}|T|^{\frac{1}{2}}\right)^{p+\frac{1}{2}} x .
$$


By Aluthge's result [1], we have

$$
\left(|T|^{\frac{1}{2}} U^{*}|T| U|T|^{\frac{1}{2}}\right)^{p+\frac{1}{2}} \geq|T|^{2\left(p+\frac{1}{2}\right)} \geq\left(|T|^{\frac{1}{2}} U|T| U^{*}|T|^{\frac{1}{2}}\right)^{p+\frac{1}{2}} .
$$

Put $\quad A=\left(|T|^{\frac{1}{2}} U^{*}|T| U|T|^{\frac{1}{2}}\right)^{p+\frac{1}{2}}, \quad B=|T|^{2\left(p+\frac{1}{2}\right)}$ and

$C=\left(|T|^{\frac{1}{2}} U|T| U^{*}|T|^{\frac{1}{2}}\right)^{p+\frac{1}{2}}$.

Then for each $x \in \mathcal{X}$, by (2) we have

$$
A x=B x=C x .
$$

We assume that $A^{n} y=B^{n} y=C^{n} y$ for each $y \in \mathcal{X}$. Since $B x \in \mathcal{X}$,

$$
\begin{aligned}
A^{n+1} x & =A^{n} A x=B^{n} A x=B^{n} B x\left(=B^{n+1} x\right) \\
& =C^{n} B x=C^{n} C x=C^{n+1} x .
\end{aligned}
$$

Hence by (3) we have $A^{n} x=B^{n} x=C^{n} x$ for every non-negative integer $n$ and $x \in \mathcal{X}$. From the above, since we have $f(A) x=f(B) x=f(C) x$ for every polynomial $f$ and $x \in \mathcal{X}$, similarly we obtain

$$
|T|^{\frac{1}{2}} U^{*}|T| U|T|^{\frac{1}{2}} x=|T|^{2} x=|T|^{\frac{1}{2}} U|T| U^{*}|T|^{\frac{1}{2}} x \in \mathcal{X} .
$$

From (4) we have

$$
\operatorname{ker} \tilde{T} \cap \mathcal{X}=\operatorname{ker}(\tilde{T})^{*} \cap \mathcal{X}=\operatorname{ker}|T| \cap \mathcal{X}=\{0\} .
$$

Since $\mathcal{X}$ is a reducing subspace of $\tilde{T}$ and $|T|$,

$$
\overline{\tilde{T}(\mathcal{X})}=\overline{(\tilde{T})^{*}(\mathcal{X})}=\mathcal{X}=\overline{|T|(\mathcal{X})}=\overline{|T|^{\frac{1}{2}}(\mathcal{X})} .
$$

Since ker $|T|=\{0\}$, from (4), we have

$$
U^{*}|T|^{\frac{1}{2}} \tilde{T} x=|T|^{\frac{3}{2}} x=U|T|^{\frac{1}{2}}(\tilde{T})^{*} x .
$$

From (5) and (6), it holds that

$$
U^{*}|T|^{\frac{1}{2}}(\mathcal{X}) \subseteq \mathcal{X} \quad \text { and } \quad U|T|^{\frac{1}{2}}(\mathcal{X}) \subseteq \mathcal{X}
$$

so that $U^{*}(\mathcal{X}) \subseteq \mathcal{X}$ and $U(\mathcal{X}) \subseteq \mathcal{X}$. Hence $\mathcal{X}$ is a reducing subspace of $|T|$ and $U$. From (5), we have

$$
|T||T|^{\frac{1}{2}} x=U|T| U^{*}|T|^{\frac{1}{2}} x .
$$

By (5), we have $|T| y=U|T| U^{*} y$ for $y \in \mathcal{X}$. Therefore, $\mathcal{X}$ is a reducing subspace of $T$ such that $T$ is normal on $\mathcal{X}$. This completes the proof. 


\section{Proofs of theorems}

In order to give proofs of the main results, we need the following theorem.

Theorem C ([6, Theorem 1.15]) For an operator $T=U|T|, T$ has a nontrivial invariant subspace if and only if so does $\tilde{T}$.

Proof of Theorem 1. If $T$ has no cyclic vectors, then $T$ has a nontrivial invariant subspaces. Hence we may assume that $T$ has a cyclic vector and is pure. By Lemma 3, all $T, \tilde{T}$ and $\hat{T}$ have dense ranges.

By Aluthge's result, $\tilde{T}$ is a semi-hyponormal operator. It follows from Lemma 4 that $\hat{T}$ is a pure hyponormal operator. Since $\sigma(\hat{T})=\sigma(T), \hat{T}$ is a hyponormal operator satisfying Theorem A. Hence $\hat{T}$ has a nontrivial invariant subspace. Therefore by Theorem $\mathrm{C}, T$ has a nontrivial invariant subspace.

A similar argument implies Theorem 2.

\section{References}

[1] Aluthge A., On p-hyponormal operator for $0<p<1$. Integr. Equat. Oper. Th. 13 (1990), 307-315.

[2] Apostol C. and Clancey K., Local resolvents of operators with one-dimensional selfcommutator. Proc. Amer. Math. Soc. 58 (1976), 158-162.

[3] Chō M., Huruya T. and Yamazaki T., Mosaic and principal functions of loghyponormal operators. J. Math. Soc. Japan 55 (2003), 255-268.

[4] Clancey K., Seminormal operators. Lecture Notes in Math. Vol. 742, SpringerVerlag, New York, 1979.

[5] Duggal B.P., p-hyponormal operators and invariant subspaces. Acta Sci. Math. (Szeged) 64 (1998), 249-257.

[6] Jung I.B., Ko E. and Pearcy C., Aluthge transforms of operators. Integr. Equat. Oper. Th. 37 (2000), 437-448.

[7] Nakamura Y., Principal functions and invariant subspaces of hyponormal operators. Hokkaido Math. J. 12 (1983), 1-9.

[ 8 ] Putnam C.R., Invariant subspaces of operators having nearly disconnected spectra. Operator theory and functional analysis, Res. Notes in Math. 38, Pitman, 1979.

[ 9] Xia D., Spectral theory of hyponormal operators. Birkhäuser Verlag, Basel (1983). 


\author{
Muneo Chō \\ Department of Mathematics \\ Kanagawa University \\ Yokohama 221-8686 \\ Japan \\ E-mail: chiyom01@kanagawa-u.ac.jp
}

Tadasi Huruya

Faculty of Education and Human Sciences Niigata University

Niigata 950-2181

Japan

E-mail: huruya@ed.niigata-u.ac.jp 